\title{
Age and recurrent stroke are related to the severity of white matter hyperintensities in lacunar infarction patients with diabetes
}

This article was published in the following Dove Press journal:

Clinical Interventions in Aging

\author{
Ling Yu ${ }^{1}$ \\ Lei Yang ${ }^{2}$ \\ Xiaoyu Zhang ${ }^{2}$ \\ Junliang Yuan ${ }^{2}$ \\ Yue $\mathrm{Li}^{2}$ \\ Shuna Yang ${ }^{2}$ \\ Hua $\mathrm{Gu}^{2}$ \\ Wenli $\mathrm{Hu}^{2}$ \\ Shan Gao' \\ 'Department of Endocrinology, Beijing \\ Chaoyang Hospital, Capital Medical \\ University, Beijing, People's Republic \\ of China; ${ }^{2}$ Department of Neurology, \\ Beijing Chaoyang Hospital, Capital \\ Medical University, Beijing, People's \\ Republic of China
}

Background and purpose: White matter hyperintensities (WMH) is identified as a marker of cerebral small vessel diseases and is a major contributor to cognitive impairment, depression, gait disturbance, and urinary incontinence. However, the risk factors for WMH in patients with type 2 diabetes mellitus (T2DM) has not been well explored. Thus, in this study, we aimed to investigate the relationship between the severity of WMH and vascular risk factors in lacunar infarction patients with T2DM.

Methods: Consecutive lacunar infarction patients with T2DM were recruited in this crosssectional study. Paraventricular WMH (P-WMH) and deep WMH (D-WMH) were separately scored by the Fazekas scale, and classified into two categories by the severity. Vascular risk factors and clinical features were compared between the mild and severe WMH. Multiple logistic regression analysis was used to determine the relationship between severity of WMH and vascular risk factors.

Results: A total of 327 participants aged 34-91 years were enrolled in this study. Compared with the patients with mild P-WMH, the patients with severe $\mathrm{P}-\mathrm{WMH}$ had higher age $(P=0.031)$, higher proportion of hypertension $(P=0.042)$ and stroke $(P<0.001)$. Levels of TG, LDL, and HbA1c were significantly higher in patients with mild P-WMH. Compared with the patients with mild D-WMH the patients with severe D-WMH had higher age and hyperhomocysteinemia (HCY) level $(P<0.001)$, higher proportion of hyperlipidemia $(P=0.008)$, and stroke $(P<0.001)$. Multivariable logistic regression analyses showed that higher age and recurrent stroke were independently related to severe P-WMH and D-WMH in lacunar infarction patients with T2DM.

Conclusions: Age and recurrent stroke are related to the severity of P-WMH and D-WMH in lacunar infarction patients with T2DM.

Keywords: lacunar infarction, type 2 diabetes mellitus, white matter hyperintensities, leukoaraiosis

\section{Introduction}

White matter hyperintensities (WMH) or leukoaraiosis are areas of hyperintense on T2-weighted sequences and fluid-attenuated inversion recovery (FLAIR), isointense or hypointense on T1-weighted sequences with respect to normal brain. ${ }^{1} \mathrm{WMH}$ is considered as the main imaging sign of cerebral small vessel diseases (CSVD) which was classified into paraventricular WMH (P-WMH) and deep WMH (D-WMH) according to the locations. A lot of studies have demonstrated that WMH was associated with cognitive decline, depression, gait disturbance and increased stroke risk. ${ }^{2}$ However, the risk factor and pathogenesis of WMH is not well known.

Currently, a review by del Bene et $\mathrm{al}^{3}$ highlights more firmly the existence of a relation between DM and both presence and severity of WMH. Interestingly, some 
studies found that the risk factors of $\mathrm{WMH}$ in subjects without T2DM were different from that in subjects with T2DM. Previous studies found that age and hypertension were independent risk factors for WMH in community populations. However, some studies found that hypertension was not related to $\mathrm{WMH}$ in T2DM patients. The results of a cross-sectional study by Van Harten et $\mathrm{al}^{4}$ showed that there were no statistical differences in WMH scores between hypertensive and non-hypertensive patients with T2DM. The study by de Bresser et $\mathrm{al}^{7}$ showed that neither hypertension nor arterial blood pressure level was related to WMH volume in elderly patients with T2DM. In addition, previous studies showed that the correlation between metabolic indicators and $\mathrm{WMH}$ was also inconsistent in patients with diabetes. A cross-sectional study investigated the associations of WMH with cognitive function, metabolic and vascular risk factors in 178 elderly patients with T2DM. They found that glycoalbumin/glycosylated hemoglobin was independently associated with WMH volumes. However, Murray et $\mathrm{al}^{5}$ found the glycosylated hemoglobin level was not associated with WMH volume in a T2DM population.

Exploring the risk factors of $\mathrm{WMH}$ is important for further treatment. To our knowledge, studies on the WMH severity and vascular risk factors in patients with T2DM are rare, and the conclusion is consistent. Therefore, we aimed to investigate the relationship between the severity of WMH and vascular risk factors in lacunar infarction patients with T2DM.

\section{Methods}

\section{Study subjects}

We retrospectively recruited the study subjects in the neurology department of Beijing Chaoyang Hospital affiliated to Capital Medical University from January 2011 to May 2015. Consecutive lacunar infarction patients were identified and collected if they had a history of T2DM.

Acute lacunar infarction was defined as an acute round or ovoid lesion of increased signal on axial diffusion-weighted imaging (DWI) $\leq 20 \mathrm{~mm}$ and coronal fluid attenuated inversion recovery (FLAIR) $\leq 20 \mathrm{~mm}$ in the distribution of a small penetrating artery (including pons). ${ }^{6}$

Patients were enrolled in the study if they met the following criteria: 1) admitted within 1 week after stroke onset; and 2) agreed to participate in this study. Patients with a definite cardioembolic source (eg, atrial fibrillation, recent myocardial infarction, dilated cardiomyopathy, valvular heart disease, or infectious endocarditis) or ipsilateral extracranial carotid stenosis were excluded.
The study was approved by the ethics committee of Beijing Chaoyang Hospital and performed in accordance with the Declaration of Helsinki. All subjects provided written informed consent.

\section{Demographic and clinical assessments}

All patients underwent a laboratory test, including aspartate aminotransferase, alanine aminotransferase, urea nitrogen (BUN), creatinine (Cr), uric acid (Uri), creatine kinase (CK), glycosylated hemoglobin (HbA1c), blood-glucose (Glu), low density lipoprotein cholesterol (LDL), high density lipoprotein cholesterol (HDL), triglycerides (TG) and hyperhomocysteinemia (HCY). All patients received cardiac evaluation (including electrocardiogram and heart ultrasound).

Demographic features and risk factors were recorded, including hypertension (defined as receiving medication for hypertension or blood pressure $>140 / 90 \mathrm{mmHg}$ on repeated measurements), diabetes mellitus (defined as receiving medication for diabetes mellitus or diagnosed at discharge), hyperlipidemia (defined as receiving cholesterol reducing agents or low-density lipoprotein cholesterol $\geq 2.6 \mathrm{mmol} / \mathrm{L}$ at the time of admission), current cigarette smoking, history of stroke, and history of coronary heart disease. National Institutes of Health Stroke Scale (NIHSS) score was measured at the time of admission and discharge.

\section{Assessments of WMH}

Brain MRI scans were performed within 1 week after stroke onset, including DWI, fluid-attenuated inversion recovery (coronal) and MRA. Brain MRI was performed with a $1.5 \mathrm{~T}$ scanner (Sigma Horizon LX, GE Healthcare, Piscataway, NJ, USA) or a 3.0 T MR scanner (Siemens, Erlangen, Germany). CTA was performed on a dual-source CT scanner (Somatom Definition; Siemens Medical Solutions, Forchheim, Germany).

P-WMH and D-WMH were separately scored by Fazekas scale. ${ }^{7}$ Participants were divided into two groups according to Fazekas scores: a "mild group," for those with a P-WMH/ D-WMH Fazekas score of 0-1 and a "severe group," for those with a PVL/DWMH Fazekas score of 2 and 3 (Figures 1 and 2).

Two investigators (Xiaoyu Zhang, Shuna Yang) blinded to clinical information, independently reviewed the MRI images and assessed the severity of WMH. In cases of discrepancy, the third investigator (Hua Gu, neuroradiologist) made the final decision. The intra-rater agreement for the rating of CSVD was assessed with a random sample of 80 individuals with a month-long interval between the first 

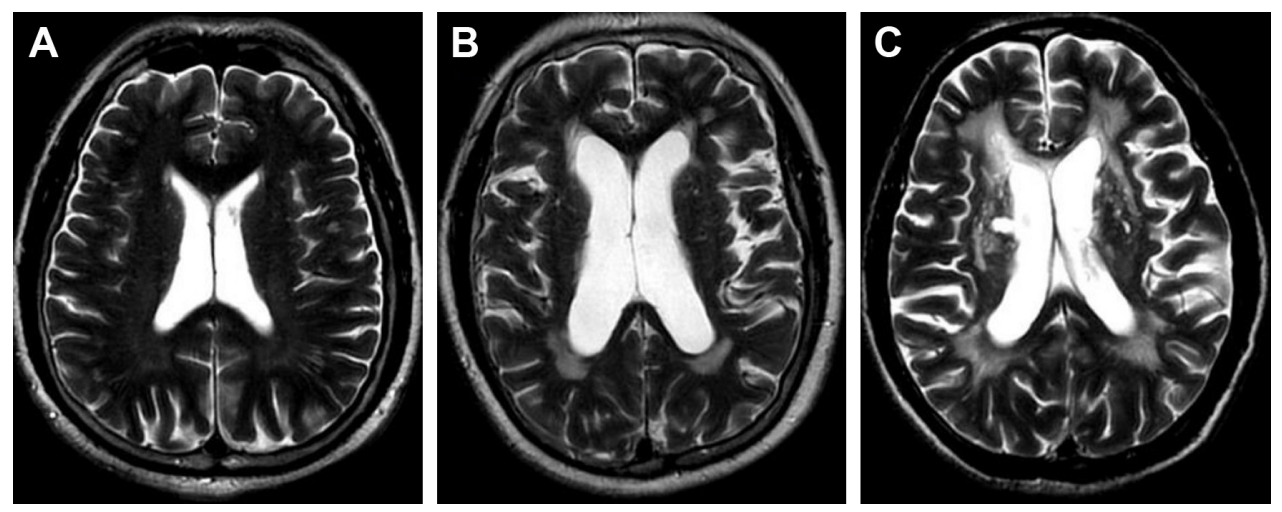

Figure I Different scores of $\mathrm{P}-\mathrm{WMH}$.

Notes: (A) One point = "caps" or pencil-thin lining; (B) two points = smooth "halo"; (C) three points = irregular P-WMH extending into the deep white matter.

Abbreviation: $\mathrm{P}-\mathrm{WMH}$, paraventricular white matter hyperintensities.

and second readings. Random scans of 80 individuals were independently examined by two experienced neurologists who were blinded to each other's readings. The $\mathrm{k}$ statistics of intra-rater and interrater agreement were 0.70 or above, which indicated good reliability. Disagreements were resolved by discussing with other co-authors.

\section{Statistical analysis}

Continuous variables were presented as mean with SD for normally distributed variables and as the median (interquartile range) for non-normal distributed variables. Independentsamples $t$-test was used for comparison of continuous variables with both normal distribution and homogeneity of variance; otherwise, the Mann-Whitney U test was used. Categorical variables were compared using Chi-square. Binary logistic regression analysis was used to adjust for age, sex, laboratory indexes, and other vascular risk factors. We adopted the severity of P-WMH and D-WMH as dependent variables and general clinical characteristics including age, sex, vascular risk factors (hypertension, hyperlipidemia, history of CAD, history of stroke, smoking), and laboratory indexes (TG, LDL, HDL, HbA1c, glucose, HCY, BUN, Cr and Uri) as explanatory variables. And then we used logistic regression analysis to determine independent predictors for WMH. The significant indicators tested by Chi-square, Independent-samples $t$-test and Mann-Whitney $\mathrm{U}$ test were put into the logistic regression model. Statistical analyses were performed using SPSS version 20.0 (IBM Corporation, Armonk, NY, USA). $P$-values of $<0.05$ were considered statistically significant.

\section{Results}

\section{General subjects' characteristics}

During the study period, a total of 1,004 acute lacunar infarction patients were identified, and 327 of them had a history of T2DM. None of them had a definite cardioembolic source or ipsilateral extracranial carotid stenosis. Therefore, 327 patients were enrolled in this study with 215 (65.7\%) males and a mean age of $62.94 \pm 11.86$ years (range, $34-91$ years).
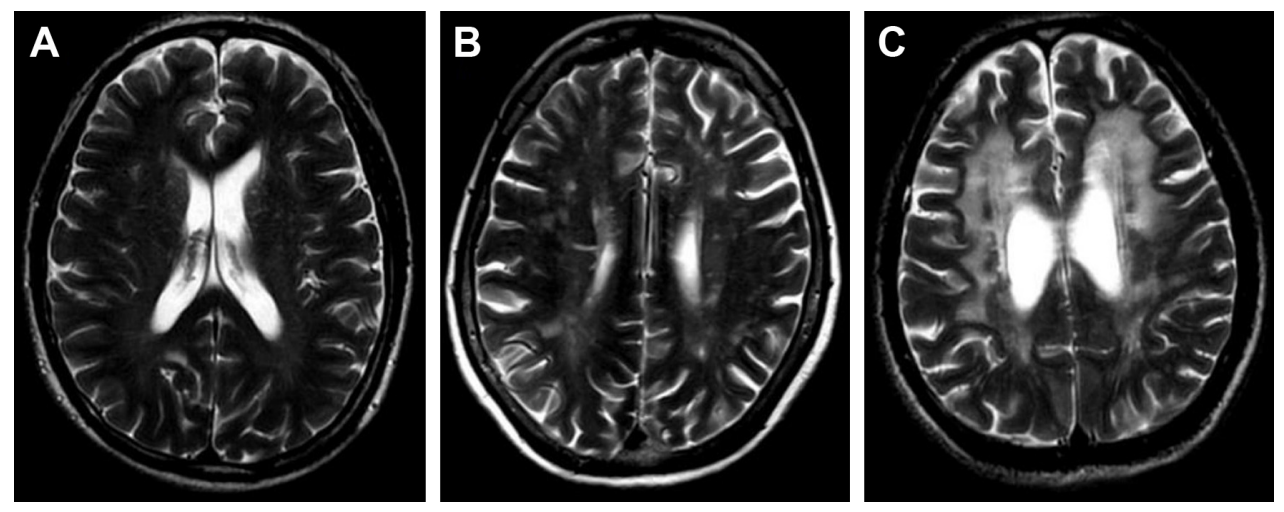

Figure 2 Different scores of D-WMH.

Notes: $(\mathbf{A})$ One point $=$ punctate foci; $(\mathbf{B})$ two points $=$ beginning confluence of foci; $(\mathbf{C})$ three points $=$ large confluence areas.

Abbreviation: D-WMH, deep white matter hyperintensities. 
Risk factors included hypertension in 250 patients $(76.5 \%)$, hyperlipidemia in 84 (25.7\%), and cigarette smoking in 165 (50.5\%). A total of 104 patients $(31.8 \%)$ had a history of stroke and $55(16.8 \%)$ had a history of coronary heart disease.

Among the 327 enrolled patients, 175 (53.5\%) participants had mild P-WMH, 152 (46.5\%) participants had severe P-WMH, 206 (63\%) participants had mild D-WMH and 121 (37\%) participants had severe D-WMH.

Detail clinical and demographic features of the participants in each group are presented in Table 1.

\section{Characteristics of patients according to the severity of P-WMH}

General clinical characteristics of the two groups stratified by the severity of P-WMH are presented in Table 1.

Compared to the mild P-WMH group, the severe $\mathrm{P}-\mathrm{WMH}$ group had higher age $(P=0.031)$, higher proportion of hypertension $(P=0.042)$ and stroke $(P<0.001)$. Whereas, the proportion of smoking was higher in patients with mild $\mathrm{P}-\mathrm{WMH}$ than patients with severe $\mathrm{P}-\mathrm{WMH}$.

Levels of HDL and HCY was significantly higher in patients with severe P-WMH. On the contrary, levels of TG, LDL, and HbAlc were significantly higher in patients with mild P-WMH.

Binary logistic regression analyses showed that higher age and the recurrent stroke were independently associated with severe degree of P-WMH after adjusting for sex and vascular risk factors. These statistical results are displayed in Table 2.

\section{Characteristics of patients according to the scale of D-WMH}

General clinical characteristics of the two groups stratified by the severity of D-WMH are presented in Table 1. Compared to the mild D-WMH group, the severe D-WMH group also had higher age $(P<0.001)$, and higher proportion of hyperlipidemia $(P=0.008)$ and stroke $(P<0.001)$. More patients had a smoking history in the mild D-WMH group.

Levels of HCY was significantly higher in patients with severe D-WMH. Levels of HbA1c were significantly higher in patients with mild D-WMH.

Binary logistic regression analyses showed higher age and the recurrent stroke were also independently associated with the severity of D-WMH after adjusting for sex and vascular risk factors. These statistical results are displayed in Table 3.

\section{Discussion}

To the best of our knowledge, this is the first study mainly focusing on exploring the risk factors for WMH of different brain areas in lacunar infarction patients with T2DM. Results showed that age and recurrent stroke are related to

Table I Characteristics of lacunar infarction patients with DM according to the scale of P-WMH and D-WMH

\begin{tabular}{|c|c|c|c|c|c|c|}
\hline \multirow[t]{2}{*}{ Clinical features } & \multicolumn{2}{|c|}{$\begin{array}{l}\text { Paraventricular white matter } \\
\text { hyperintensities }\end{array}$} & \multirow[t]{2}{*}{$P$-value } & \multicolumn{2}{|c|}{$\begin{array}{l}\text { Deep white matter } \\
\text { hyperintensities }\end{array}$} & \multirow[t]{2}{*}{$P$-value } \\
\hline & (175, mild) & (152, severe) & & $\leq \mathrm{I}(206, \mathrm{mild})$ & $\geq 2(121$, severe $)$ & \\
\hline Age, ${ }^{a}$ years & $59.9 \pm 8.4$ & $67.6 \pm 9.6$ & 0.031 & $61.3 \pm 9$ & $67.3 \pm 10$ & $<0.001$ \\
\hline Male, no (\%) & $126(72 \%)$ & $84(55.3 \%)$ & 0.002 & 143 (69.4\%) & $67(55.4 \%)$ & 0.011 \\
\hline Hypertension, no (\%) & $126(72 \%)$ & $124(81.6 \%)$ & 0.042 & $|5|(73.3 \%)$ & $99(81.8 \%)$ & 0.08 \\
\hline Hyperlipidemia, no (\%) & $49(28 \%)$ & $35(23 \%)$ & 0.305 & $63(30.6 \%)$ & 21 (I7.4\%) & 0.008 \\
\hline History of CAD, no (\%) & $28(16 \%)$ & 27 (I7.8\%) & $0.67 \mathrm{I}$ & 32 (I5.5\%) & $23(19 \%)$ & 0.417 \\
\hline History of stroke, no (\%) & $29(16.6 \%)$ & $74(48.7 \%)$ & $<0.001$ & 41 (19.9\%) & $62(51.2 \%)$ & $<0.001$ \\
\hline Smoking, no (\%) & $108(61.7 \%)$ & 57 (37.5\%) & $<0.001$ & 114 (55.3\%) & $5 I(42.1 \%)$ & 0.021 \\
\hline NIHSS ${ }^{b}$ & $3(1-5)$ & $3(2-5)$ & 0.157 & $3(I-5)$ & $3(2-5)$ & 0.032 \\
\hline $\mathrm{TG}^{\mathrm{b}}(\mathrm{mmol} / \mathrm{L})$ & $1.57(1.11-2.55)$ & $1.42(0.95-1.84)$ & 0.01 & $1.5(1.08-2.49)$ & I.42 (0.95-I.87) & 0.062 \\
\hline $\mathrm{LDL}^{\mathrm{a}}(\mathrm{mmol} / \mathrm{L})$ & $2.7 \pm 0.8$ & $2.5 \pm 0.7$ & 0.02 & $2.7 \pm 0.8$ & $2.56 \pm 0.75$ & 0.182 \\
\hline $\mathrm{HDL}^{\mathrm{a}}(\mathrm{mmol} / \mathrm{L})$ & $1.1 \pm 0.3$ & $1.2 \pm 0.3$ & 0.027 & $1.17 \pm 0.3$ & $1.25 \pm 0.3$ & 0.059 \\
\hline $\mathrm{HbAIc}(\%)$ & $8.4(6.9-9.9)$ & $7.5(6.7-8.8)$ & 0.01 & $8.3(7-9.6)$ & $7.4(6.5-9)$ & 0.006 \\
\hline $\mathrm{Glu}^{\mathrm{a}}(\mathrm{mmol} / \mathrm{L})$ & $9 \pm 3.5$ & $8.4 \pm 3.3$ & 0.103 & $8.9 \pm 3.6$ & $8.3 \pm 3.2$ & 0.107 \\
\hline $\mathrm{HCY}^{\mathrm{b}}$ (umol/L) & $14(13-17)$ & $15(13-19)$ & 0.022 & $14(13-17)$ & $16(13-19)$ & 0.008 \\
\hline $\mathrm{BUN}^{\mathrm{b}}(\mathrm{mmol} / \mathrm{L})$ & $4.7(3.9-6)$ & $4.8(4.1-5.7)$ & $0.81 \mathrm{I}$ & $4.8(4-5.9)$ & $4.7(4-5.7)$ & 0.456 \\
\hline $\mathrm{Cr}^{\mathrm{b}}(\mathrm{umol} / \mathrm{L})$ & 78 (66-89) & $76(62-93)$ & 0.545 & 77.7 (65.9-89) & $78.4(62.6-94.5)$ & 0.547 \\
\hline Uria (umol/L) & $295.6 \pm 82.1$ & $279.9 \pm 82.1$ & 0.086 & $284.5 \pm 74.4$ & $294.7 \pm 94$ & $0.28 I$ \\
\hline
\end{tabular}

Notes: aContinuous variables with normal distribution were expressed as mean $\pm \mathrm{SD}$; ${ }^{\mathrm{b}}$ Continuous variables with non-normal distribution were expressed as the median (interquartile range).

Abbreviations: DM, diabetes mellitus; P-WMH, paraventricular white matter hyperintensities; D-WMH, deep white matter hyperintensities; CAD, coronary artery atherosclerosis disease; TG, triglycerides; LDL, low-density lipoprotein; HDL, high-density lipoprotein; HbA Ic, glycosylated hemoglobin; Cr, blood creatinine; Uri, blood uric acid; HCY, homocysteinemia; BUN, blood urea nitrogen. 
Table 2 The results of binary logistic regression analyses on the association between P-WMH and risk factors in lacunar infarction patients with DM

\begin{tabular}{|l|l|l|l|}
\hline Clinical features & Odds ratio & $\mathbf{9 5 \%} \mathbf{C l}$ & P-value \\
\hline Age & 1.065 & $1.025-1.106$ & 0.001 \\
Male & 1.345 & $0.555-3.263$ & 0.512 \\
History of hypertension & 0.853 & $0.388-1.875$ & 0.692 \\
History of stroke & 5.447 & $2.659-11.159$ & $<0.00 \mathrm{I}$ \\
Smoking & 0.458 & $0.196-1.067$ & 0.07 \\
TG & 0.867 & $0.669-1.124$ & 0.282 \\
LDL & 0.77 & $0.488-1.216$ & 0.263 \\
HDL & 0.921 & $0.327-2.596$ & 0.921 \\
HbAlc & 0.856 & $0.705-1.039$ & 0.116 \\
HCY & 1.063 & $0.989-1.142$ & 0.082 \\
\hline
\end{tabular}

Abbreviations: P-WMH, paraventricular white matter hyperintensities; DM, diabetes mellitus; TG, triglycerides; LDL, low-density lipoprotein; HDL, high-density lipoprotein; HbAlc, glycosylated hemoglobin; $\mathrm{HCY}$, hyperhomocysteinemia.

the severity of P-WMH and D-WMH in lacunar infarction patients with T2DM.

WMH and lacunar infarction are the main imaging signs of CSVD. The prevalence of WMH is greater in individuals with vascular risk factors, notably hypertension ${ }^{8-11}$ and diabetes mellitus. Some studies have demonstrated that anatomical brain abnormalities were more often observed in adults with T2DM, including increased presence of subcortical infarcts, ${ }^{12}$ and white matter lesions, ${ }^{4}$ than healthy controls.

It is well known that hypertension is considered the main risk factor for WMH and lacunar infarction. More and more researchers have found that there is a relationship between T2DM and both presence and severity of WMH. Two crosssectional studies ${ }^{13,14}$ showed that there was no association between DM and WMH. Another two prospective studies found a significant association between T2DM and the progression of $\mathrm{WMH}$. Taylor et $\mathrm{al}^{15}$ found that patients with T2DM had greater WMH progression in 113 patients aged over 60 years old through a 2 -year follow-up. They concluded

Table 3 Multivariable models of the association between $\mathrm{D}-\mathrm{WMH}$ and risk factors in lacunar infarction patients with DM

\begin{tabular}{|l|l|l|l|}
\hline Clinical features & Odd ratios & $\mathbf{9 5 \%} \mathbf{C l}$ & P-value \\
\hline Age & 1.05 & $1.013-1.087$ & 0.008 \\
Male & 1.579 & $0.666-3.741$ & 0.3 \\
Hypertension & 1.4 & $0.646-3.038$ & 0.394 \\
Hyperlipidemia & 0.376 & $0.164-0.862$ & 0.021 \\
History of stroke & 5.41 & $2.664-10.986$ & $<0.00 \mathrm{I}$ \\
Smoking & 0.745 & $0.327-1.695$ & 0.483 \\
TG & 1.041 & $0.815-1.329$ & 0.749 \\
HDL & 1.269 & $0.463-3.473$ & 0.644 \\
HbAlc & 0.865 & $0.715-1.045$ & 0.133 \\
HCY & 1.069 & $0.997-I .147$ & 0.062 \\
\hline
\end{tabular}

Abbreviations: D-WMH, deep white matter hyperintensities; DM, diabetes mellitus; TG, triglycerides; HDL, high-density lipoprotein; HbAlc, glycosylated hemoglobin; HCY, hyperhomocysteinemia. that diabetes is a potentially important contributor to lesion volume progression. In the LADIS study, Gouw et $\mathrm{al}^{16}$ followed 396 elderly patients for 3 years. They found previous stroke, diabetes, and blood glucose were risk factors for WMH progression. In contrast, one cross-sectional study ${ }^{13}$ and two longitudinal studies ${ }^{17,18}$ failed to find any difference in WMH progression between DM and non-DM patients. In the PROSPER study, van Elderen et $a{ }^{17}$ followed 527 elderly patients for 3 years. There was no statistical difference in the progression of WMH volume or infarctions between DM and non-DM groups. A review by del Bene et $\mathrm{al}^{3}$ highlights more firmly than previously reported the existence of a relation between DM and both presence and severity of WMH.

Interestingly, when study populations were limited to T2DM, the results changed. And the studies on risk factors for WMH in T2DM were not enough. Therefore, our study population was limited to patients with T2DM and then we attempted to search for the factors affecting severity of WMH. Vascular risk factors, such as hypertension, are considered the main risk factor for WMH. In a cross-sectional study, van Harten et $\mathrm{al}^{4}$ showed that WMH scores did not differ between hypertensive and non-hypertensive patients with T2DM. Manschot et $\mathrm{al}^{12}$ also found that hypertension was not related to WMH severity. In a prospective study of elderly patients with T2DM, de Bresser et al ${ }^{18}$ showed that neither hypertension nor arterial blood pressure significantly affected WMH volume. Our study focused on lacunar infarction patients with T2DM, and found some traditional vascular risk factors had significant association with WMH. Our patients with severe P-WMH had higher age, often had a history of hypertension and stroke. Patients with severe $\mathrm{D}-\mathrm{WMH}$ also had higher age and were more likely to have a history of stroke. Hypertension was not related to the severity of D-WMH. However, binary logistic regression analyses showed that only older age and recurrent stroke independently associated with severe WMH, not hypertension, which may be caused by our study population. Our study subjects were patients with lacunar infarction and lacunar infarction was well known to be caused by hypertensive lipohyalinosis. This shows that our lacunar infarction patients have hypertension background. The different study population may explain our result: hypertension is not an independent risk factor.

There may be significant association between some clinical features and the severity of WMH although the results have been variable. In a study of subjects without T2DM, Murray et $\mathrm{al}^{5}$ showed that the HbA1c level was positively correlated with WMH. However, in another two studies, ${ }^{12,18}$ the $\mathrm{HbA1}$ c level was not associated with WMH volume. A crosssectional study reported by Manschot et al ${ }^{12}$ found T2DM 
was associated with D-WMH. Within the type 2 diabetic group, there was a modest association WMH with $\mathrm{HbA1c}$ and diabetes duration. However, multiple logistic regression analysis showed only age had statistically significant correlation. Another cross-sectional study based on T2DM patients found no significant association between $\mathrm{HbA} 1 \mathrm{c}$ and WMH. ${ }^{19}$ However, the value of GA (glycoalbumin)/HbA1c was positively correlated to WMH. Interestingly, our result showed the level of HbA1c was higher in patients with mild $\mathrm{P}-\mathrm{WMH}$ and D-WMH. Logistic regression analyses also showed no significant associations about $\mathrm{HbA} 1 \mathrm{c}$ and $\mathrm{WMH}$ in both groups.

A cohort study based on an elderly population including non-DM subjects showed that TG and LDL have opposite effects on WMH. TG increased with a larger WMH volume, and increasing LDL was associated with a decrease in the severity of $\mathrm{WMH},{ }^{20}$ which indicate that high $\mathrm{TG}$ reflects insulin resistance and high LDL reflects nutritional status. Our study found levels of TG and LDL were higher in patients with mild $\mathrm{P}-\mathrm{WMH}$, but not in patients with severe $\mathrm{D}-\mathrm{WMH}$, which supported that the pathogenesis of $\mathrm{P}-\mathrm{WMH}$ and D-WMH might be different. Logistic regression analyses showed there were no significant associations between cholesterol and both $\mathrm{P}-\mathrm{WMH}$ and D-WMH.

It is well known that HCY is associated with endothelial dysfunction, and that high homocysteine levels in plasma are associated with WMH lesions in patients with DM. Tseng et $\mathrm{al}^{21}$ reported HCY was a risk factor for cerebral white matter lesion in stroke patients. Even mild HCY can significantly increase severity of cerebral microangiopathy. One study by Feng et $\mathrm{al}^{22}$ included 324 non-stroke patients, found HCY level associated with CSVD (defined as WMH and silent brain infarctions) more strongly than large vessel disease. In our study, levels of HCY was higher in patients with severe D-WMH. However, logistic regression analyses showed no significant associations about HCY and WMH after adjusting for confounders.

Diffusion Tensor Imaging (DTI) metrics, which can be used to provide indices of white matter axonal "integrity," tract anatomy and connectivity between brain regions. Alterations to brain function in T2DM have also been observed in blood oxygen level dependent (BOLD) functional MRI (fMRI) studies. Several studies through DTI demonstrated that microstructural white matter abnormalities may contribute to deficits in brain structure and function in adults with T2DM. ${ }^{23-25}$ Studies using innovative MRI techniques, such as diffusion tensor imaging (DTI), reported a significant association between microstructural white matter alterations and DM. ${ }^{26}$ Further studies using the sensitive and advanced imaging techniques are needed.
Our results showed only older age and recurrent stroke had significant association with severe WMH including $\mathrm{P}-\mathrm{WMH}$ and D-WMH. WMH prevalence and volume increase exponentially with age, ${ }^{11}$ and the prevalence is greater in individuals with higher levels of and longer exposure to various vascular risk factors, notably hypertension and diabetes mellitus. It has been shown that white matter changes are more extensive in patients with lacunar infarct than in those with non-lacunar ischemic stroke. ${ }^{27}$ Rost et al ${ }^{28}$ analyzed two cohorts of patients, found greater WMH burden was associated with small vessel stroke compared with other ischemic stroke subtypes. Our study is consistent with the results of the aforementioned study.

Our study subjects were T2DM patients with lacunar infarction. Lacunar infarction is well known to be caused by hypertensive lipohyalinosis. Hypertension is considered the main risk factor for leukoaraiosis. Currently, more and more evidence has shown that diabetes is also associated with leukoaraiosis. A study including 122 lacunar patients by Arboix et $\mathrm{al}^{29}$ found hypertension and diabetes were significant factors related to recurrent lacunar infarction. Patients with hypertension and diabetes may have metabolic abnormalities (dyslipidemia and hyperhomocysteinemia). Previous studies have shown dyslipidemia and hyperhomocysteinemia are associated with leukoaraiosis. However, our study showed metabolic markers were not independent predictors for severity of WMH. Metabolic indicators may have synergistic effects with diabetes. This could explain our result: metabolic indicators are not independent risk factors of WMH. Only lacunar stroke patients were included in our study which may reduce the external validity of our study.

WMH was demonstrated to be associated with cognitive decline. Blanco-Rojas et $\mathrm{al}^{30}$ found that more than half of patients with a first-ever lacunar stroke and without cognitive impairment showed minor neuropsychological alterations. These minor disturbances were mainly related to the presence of clinically silent lacunar infarcts, without any relationship to cognitive impairment with $\mathrm{WMH}$ at this early stage of cerebral small vessel disease. The follow-up of these patients may be interesting to assess whether neuropsychological disturbances are predictive factors of post-stroke dementia in the mid-term, which should be explored in future studies. In the present study, we mainly focused on the relation between risk factors and $\mathrm{WMH}$ in patients with diabetes. Exploring risk factors for $\mathrm{WMH}$ has important clinical significance for further treatment. Our results showed that age and recurrent stroke are related to the severity of P-WMH and D-WMH in lacunar infarction patients with T2DM. WHM had similar risk factors with lacunar infarction, and age is an 
uncontrollable risk factor. Therefore, prevention of stroke is particularly important.

There are several limitations in our study. First, our study was conducted in a single center. Second, this is a cross-sectional study which prevents us from making causal inference. Third, our patients received $1.5 \mathrm{~T}$ or $3 \mathrm{~T} \mathrm{MRI}$, and this may increase the variability of the WMH assessment. Fourth, our research did not measure cognitive function, depression or gait disturbance, so we could not observe the relations between $\mathrm{WMH}$ and clinical functions.

\section{Conclusion}

In the present study, we explored the risk factors of $\mathrm{MWH}$ on different brain areas in lacunar infarction patients with T2DM. The results suggest that age and recurrent stroke may be related to the severity of WMH in lacunar infarction patients with T2DM. This conclusion may only be applicable to lacunar infarction patients with T2DM.

\section{Acknowledgment}

We are grateful to Dr Wei Qin for help in the implementation of this study.

\section{Disclosure}

The authors report no conflicts of interest in this work.

\section{References}

1. Pantoni L. Cerebral small vessel disease: from pathogenesis and clinical characteristics to therapeutic challenges. Lancet Neurol. 2010; 9(7):689-701.

2. Moran C, Phan TG, Srikanth VK. Cerebral small vessel disease: a review of clinical, radiological, and histopathological phenotypes. Int J Stroke. 2012;7(1):36-46.

3. del Bene A, Ciolli L, Borgheresi L, Poggesi A, Inzitari D, Pantoni L. Is type 2 diabetes related to leukoaraiosis? An updated review. Acta Neurol Scand. 2015;132(3):147-155.

4. van Harten B, Oosterman JM, Potter van Loon BJ, Scheltens P, Weinstein HC. Brain lesions on MRI in elderly patients with type 2 diabetes mellitus. Eur Neurol. 2007;57(2):70-74.

5. Murray AD, Staff RT, Shenkin SD, Deary IJ, Starr JM, Whalley LJ. Brain white matter hyperintensities: relative importance of vascular risk factors in nondemented elderly people. Radiology. 2005;237(1): 251-257.

6. Fisher CM. Lacunar strokes and infarcts: a review. Neurology. 1982;32(8):871-876.

7. Fazekas F, Chawluk JB, Alavi A, Hurtig HI, Zimmerman RA. MR signal abnormalities at $1.5 \mathrm{~T}$ in Alzheimer's dementia and normal aging. $\mathrm{Am}$ J Roentgenol. 1987;149(2):351-356.

8. Dufouil C, de Kersaint-Gilly A, Besançon V, et al. Longitudinal study of blood pressure and white matter hyperintensities: the EVA MRI Cohort. Neurology. 2001;56(7):921-926.

9. Allan CL, Zsoldos E, Filippini N, et al. Lifetime hypertension as a predictor of brain structure in older adults: cohort study with a 28-year follow-up. Br J Psychiatry. 2015;206(4):308-315.

10. Schmidt R, Fazekas F, Kleinert G, et al. Magnetic resonance imaging signal hyperintensities in the deep and subcortical white matter. A comparative study between stroke patients and normal volunteers. Arch Neurol. 1992;49(8):825-827.
11. Liao D, Cooper L, Cai J, et al. The prevalence and severity of white matter lesions, their relationship with age, ethnicity, gender, and cardiovascular disease risk factors: the ARIC Study. Neuroepidemiology. 1997; 16(3):149-162.

12. Manschot SM, Brands AM, van der Grond J, et al. Brain magnetic resonance imaging correlates of impaired cognition in patients with type 2 diabetes. Diabetes. 2006;55(4):1106-1113.

13. Schmidt R, Launer LJ, Nilsson LG, et al. Magnetic resonance imaging of the brain in diabetes: the Cardiovascular Determinants of Dementia (CASCADE) Study. Diabetes. 2004;53(3):687-692.

14. den Heijer T, Vermeer SE, van Dijk EJ, et al. Type 2 diabetes and atrophy of medial temporal lobe structures on brain MRI. Diabetologia. 2003;46(12):1604-1610.

15. Taylor WD, Macfall JR, Provenzale JM, et al. Serial MR imaging of volumes of hyperintense white matter lesions in elderly patients: correlation with vascular risk factors. Am J Roentgenol. 2003;181(2):571-576.

16. Gouw AA, van der Flier WM, Fazekas F, et al. Progression of white matter hyperintensities and incidence of new lacunes over a 3-year period: the Leukoaraiosis and Disability Study. Stroke. 2008;39(5): $1414-1420$

17. van Elderen SG, de Roos A, de Craen AJ, et al. Progression of brain atrophy and cognitive decline in diabetes mellitus: a 3-year follow-up. Neurology. 2010;75(11):997-1002.

18. de Bresser J, Tiehuis AM, van den Berg E, et al. Progression of cerebral atrophy and white matter hyperintensities in patients with type 2 diabetes. Diabetes Care. 2010;33(6):1309-1314.

19. Tamura Y, Kimbara Y, Yamaoka T, et al. White matter hyperintensity in elderly patients with diabetes mellitus is associated with cognitive impairment, functional disability, and a high glycoalbumin/ glycohemoglobin ratio. Front Aging Neurosci. 2017;9:220.

20. Schilling S, Tzourio C, Dufouil C, et al. Plasma lipids and cerebral small vessel disease. Neurology. 2014;83(20):1844-1852.

21. Tseng YL, Chang YY, Liu JS, Su CS, Lai SL, Lan MY. Association of plasma homocysteine concentration with cerebral white matter hyperintensity on magnetic resonance images in stroke patients. J Neurol Sci. 2009;284(1-2):36-39.

22. Feng C, Bai X, Xu Y, Hua T, Huang J, Liu XY. Hyperhomocysteinemia associates with small vessel disease more closely than large vessel disease. Int J Med Sci. 2013;10(4):408-412.

23. Reijmer YD, Brundel M, de Bresser J, et al. Microstructural white matter abnormalities and cognitive functioning in type 2 diabetes: a diffusion tensor imaging study. Diabetes Care. 2013;36(1):137-144.

24. Hsu JL, Chen YL, Leu JG, et al. Microstructural white matter abnormalities in type 2 diabetes mellitus: a diffusion tensor imaging study. Neuroimage. 2012;59(2):1098-1105.

25. Hoogenboom WS, Marder TJ, Flores VL, et al. Cerebral white matter integrity and resting-state functional connectivity in middle-aged patients with type 2 diabetes. Diabetes. 2014;63(2):728-738.

26. Lucatelli P, Montisci R, Sanfilippo R, et al. Is there an association between leukoaraiosis volume and diabetes? J Neuroradiol. 2016;43(4): 273-279.

27. Arboix A, Blanco-Rojas L, Martí-Vilalta JL. Advancements in understanding the mechanisms of symptomatic lacunar ischemic stroke: translation of knowledge to prevention strategies. Expert Rev Neurother. 2014;14(3):261-276.

28. Rost NS, Rahman RM, Biffi A, et al. White matter hyperintensity volume is increased in small vessel stroke subtypes. Neurology. 2010; 75(19):1670-1677.

29. Arboix A, Font A, Garro C, García-Eroles L, Comes E, Massons J. Recurrent lacunar infarction following a previous lacunar stroke: a clinical study of 122 patients. J Neurol Neurosurg Psychiatry. 2007;78(12): 1392-1394.

30. Blanco-Rojas L, Arboix A, Canovas D, Grau-Olivares M, Oliva Morera JC, Parra O. Cognitive profile in patients with a first-ever lacunar infarct with and without silent lacunes: a comparative study. BMC Neurol. 2013;13:203. 


\section{Publish your work in this journal}

Clinical Interventions in Aging is an international, peer-reviewed journal focusing on evidence-based reports on the value or lack thereof of treatments intended to prevent or delay the onset of maladaptive correlates of aging in human beings. This journal is indexed on PubMed Central, MedLine,

CAS, Scopus and the Elsevier Bibliographic databases. The manuscript management system is completely online and includes a very quick and fair peer-review system, which is all easy to use. Visit http://www.dovepress. com/testimonials.php to read real quotes from published authors. 